\title{
Spontaneous nuclear polarization of atomic hydrogen at low magnetic field
}

\author{
V. G. Luppov ${ }^{\text {a) }}$ and T. Roser ${ }^{\text {b) }}$ \\ Randall Laboratory of Physics, University of Michigan, Ann Arbor, Michigan 48109-1120
}

(Received 23 January 1992; accepted for publication 22 June 1992)

The known spin-exchange rates of atomic hydrogen lead to fast spontaneous buildup of high nuclear polarization at low magnetic field. We propose to use the nuclear polarized atomic hydrogen gas to produce an intense ultracold nuclear polarized beam of atomic hydrogen.

Spontaneous nuclear polarization of electron polarized atomic hydrogen in a high magnetic field due to a preferential recombination of the $a$-state atoms was predicted by Statt and Berlinsky ${ }^{1}$ and was first observed by Cline et $a l^{2}$ $a, b, c$, and $d$ label the four hydrogen hyperfine statcs with increasing energy. After about 15 min $99.8 \%$ nuclear polarization has been achieved in a magnetic field of $8 \mathrm{~T}$ by Sprik et $a l^{3}$ Katunin et $a l^{4}$ accelerated the buildup of nuclear spin polarization in atomic hydrogen by driving the $a \rightarrow c$ hyperfine transition. The dominant fast spin-exchange process $a+c \rightarrow d+b$ further increased the nuclear polarization. A polarization of up to $95 \%$ was reached after pumping the $a \rightarrow c$ transition for $5 \mathrm{~min}$.

Spontaneous buildup of high nuclear polarization of electron-spin polarized atomic hydrogen can be achieved due to several fast spin-exchange processes between the two hyperfine states $c$ and $d$ at low magnetic field. At a magnetic field below about $0.5 \mathrm{~T}$ the following spinexchange processes are dominant ${ }^{5}: c+c \rightarrow a+a, c+c \rightarrow b$ $+d, c+c \rightarrow a+c$. The resulting high-field seekers ( $a$ and $b$ atoms) will rapidly escape from the low magnetic region in the presence of a proper field gradient. The loss of $c$-state atoms through spin exchange will result in a fast spontaneous buildup of nuclear polarization. This was confirmed by experiments with low-field seekers ( $c$ and $d$ states) trapped in a static magnetic trap. ${ }^{6}$ However, the characteristic time for the buildup of nuclear polarization to at least $90 \%$ was still about $3 \mathrm{~s}$ at the low density of $10^{12}$ atoms $/ \mathrm{cm}^{3}$.

We propose here to use a superfluid helium-coated confining cell placed in a low magnetic field ( $\leqslant 0.1 T$ ) with a proper gradient to accumulate a high density of low-field seekers. At a density of $10^{15}$ atoms $/ \mathrm{cm}^{3}$ the nuclear polarization reaches $90 \%$ in only about $40 \mathrm{~ms}$. The lifetime of the remaining $d$-state population is about 100 times longer. ${ }^{5}$ The sample of nuclear polarized atomic hydrogen gas can then be used to produce an ultracold intense nuclear polarized atomic hydrogen beam.

A possible scheme is shown in Fig. 1. The confining cell is placed in a magnetic field gradient with the exit aperture located at the low-field side. $c$ - and $d$-state atoms are both fed at a rate $f$ into the low-field region. The rate

\footnotetext{
- 'Permanent address: Laboratory of High Energy, Joint Institute for Nuclear Research, P.O. Box 79, Head Post Office, Moscow, Russia.

b'Permanent address: AGS Department, Brookhaven National Laboratory, Upton, NY 11973.
}

equations for the four hyperfine states in the low magnetic field region can then be written as follows:

$$
\begin{aligned}
\dot{n}_{a}= & R_{a}-2 K_{a a} n_{a}^{2}-K_{a b} n_{a} n_{b}-K_{a c} n_{a} n_{c}-K_{a d} n_{a} n_{d}-e_{H} n_{a}, \\
\dot{n}_{b}= & R_{b}-K_{a b} n_{a} n_{b}-K_{b c} n_{b} n_{c}-K_{b d} n_{b} n_{d}-e_{H} n_{b}, \\
\dot{n}_{c}= & R_{c}-K_{a c} n_{a} n_{c}-K_{b c} n_{b} n_{c}-2 K_{c c} n_{c}^{2}-K_{c d} n_{c} n_{d} \\
& -e_{L} n_{c}+f / V_{\mathrm{eff}}, \\
\dot{n}_{d}= & R_{d}-K_{a d} n_{a} n_{d}-K_{b d} n_{b} n_{d}-K_{c d} n_{c} n_{d}-e_{L} n_{d}+f / V_{\mathrm{eff}}
\end{aligned}
$$

with

$$
\begin{gathered}
R_{i}=\sum_{\substack{\alpha, \beta, \gamma, \delta=\{a, b, c, d\} \\
a \leqslant \beta, \gamma \leqslant \delta}} G_{\alpha \beta, \gamma \delta} n_{\alpha} n_{\beta}\left(-\delta_{\alpha i}-\delta_{\beta i}+\delta_{\gamma i}+\delta_{\delta i}\right) \\
i=\{a, b, c, d\},
\end{gathered}
$$

where $n_{i}$ is the density of the hyperfine state $i, G_{\alpha \beta, \gamma \delta}$ is the rate of the two-body process (spin exchange or electronic magnetic dipolar relaxation) $\alpha+\beta \rightarrow \gamma+\delta, K_{i j}$ are the effective two-body recombination constants, $e_{H}$ and $e_{L}$ are the escape rates of high- and low-field seekers through the confining cell and exit aperture, respectively, and $V_{\text {eff }}$ is the effective confining volume for the low-field seekers. For low magnetic field $(<1 \mathrm{~T})$ the following spin-exchange processes were taken into account: $b+d \leftrightarrow a+a, c+c \leftrightarrow a$ $+a, c+c \leftrightarrow b+d, b+d \leftrightarrow a+c, c+c \leftrightarrow a+c, a+c \leftrightarrow a+a, d$ $+d \leftrightarrow a+a, \quad c+d \leftrightarrow a+b, \quad d+d \leftrightarrow a+d, \quad c+c \leftrightarrow b+b, \quad d$ $+d \leftrightarrow a+c$. Other decay processes are negligible in our case.

We present here typical results calculated for a hydrogen gas temperature of $0.35 \mathrm{~K}$, a confining cell diameter of $2.5 \mathrm{~cm}$, and a magnetic field gradient of $0.8 \mathrm{~T} / \mathrm{cm}$. The calculated magnetic field dependence of the spin-exchange and dipolar relaxation rates was taken from Ref. 5 for all processes where the magnetic potential energy was lower in the final state. The rates for the remaining processes were obtained by multiplying the inverse process by the Boltzmann factor $\exp \left[\left(E_{i}-E_{f}\right) / k T\right]$. We used the measured two-body recombination constants of Ref. 7.

Figure 2 shows the calculated ratio of $d$-state density to $c$-state density and the spontaneous nuclear polarization at zero magnetic field $\left[p=n_{d} /\left(n_{d}+n_{c}\right)\right]$ as a function of the magnetic field at the aperture location. The calculations were made for a feed rate of $10^{17}$ atoms/s with closed exit aperture. It can be seen that the polarization begins to 


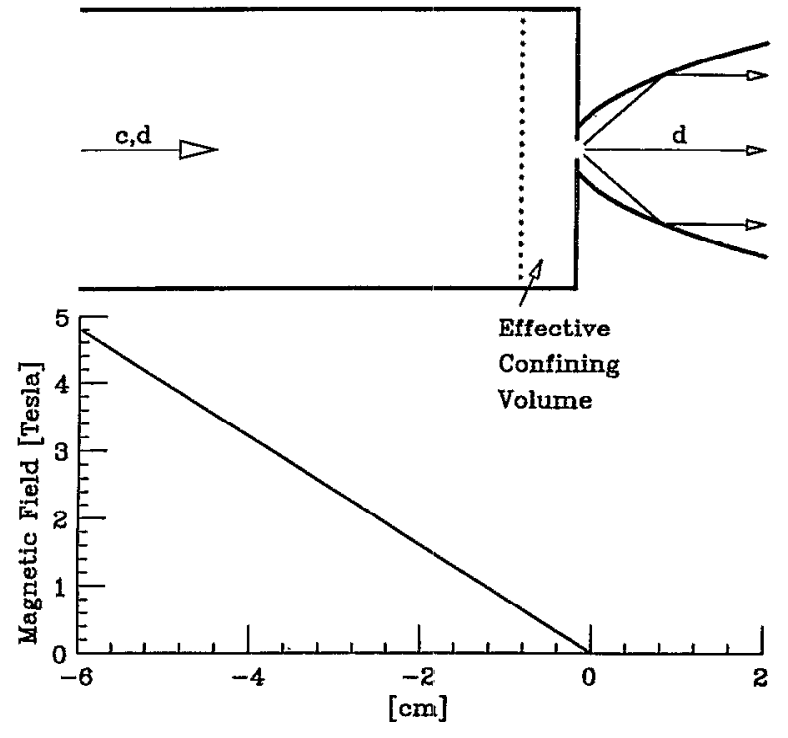

FIG. 1. Schematic layout of the superfluid helium-coated confiing cell shown relative to the magnetic field profile. The dashed line shows a focusing parabolic mirror which would reflect more than $90 \%$ of the outcoming atoms. Details are discussed in the text.

decrease at a magnetic field of $0.1 \mathrm{~T}$. At this field the spin-exchange and dipole relaxation processes that involve the $d$ state become significant. Changing the feed rate within the limits of $10^{16}-10^{18}$ atoms/s does not influence the results significantly.

Spontaneous nuclear polarization at zero magnetic field of the escaping beam of the low-field seekers calculated for various values of the magnetic field at the $2-\mathrm{mm}$ diam aperture versus the feed rate of $c$ and $d$-states atoms is shown in Fig. 3. In this case, the nuclear polarization improves with increasing feed rate because with increasing

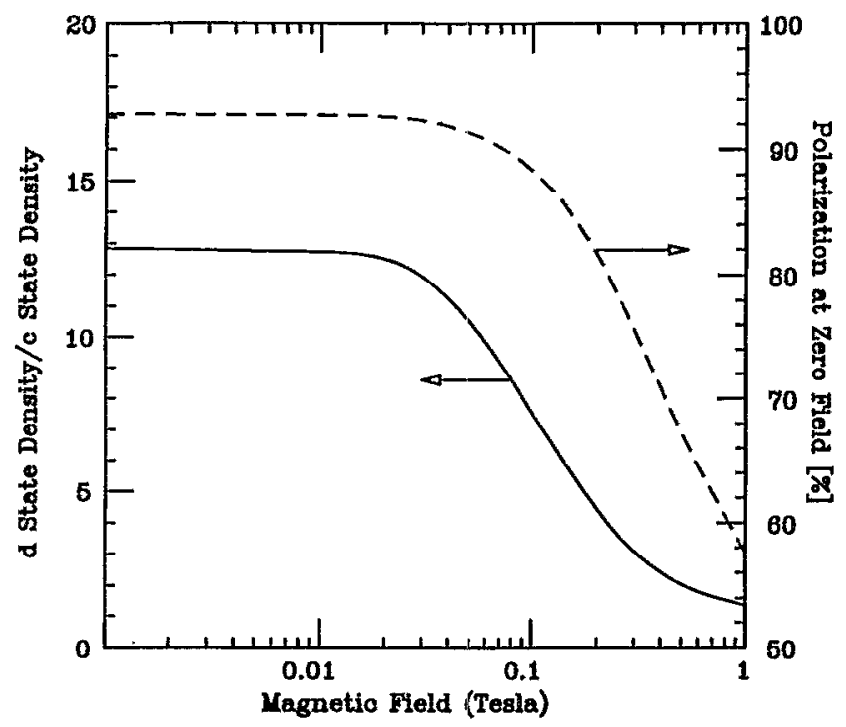

FIG. 2. Ratio of the $d$-state density to the $c$-state density and the spontaneous nuclear polarization with closed exit aperture as a function of the magnetic field.

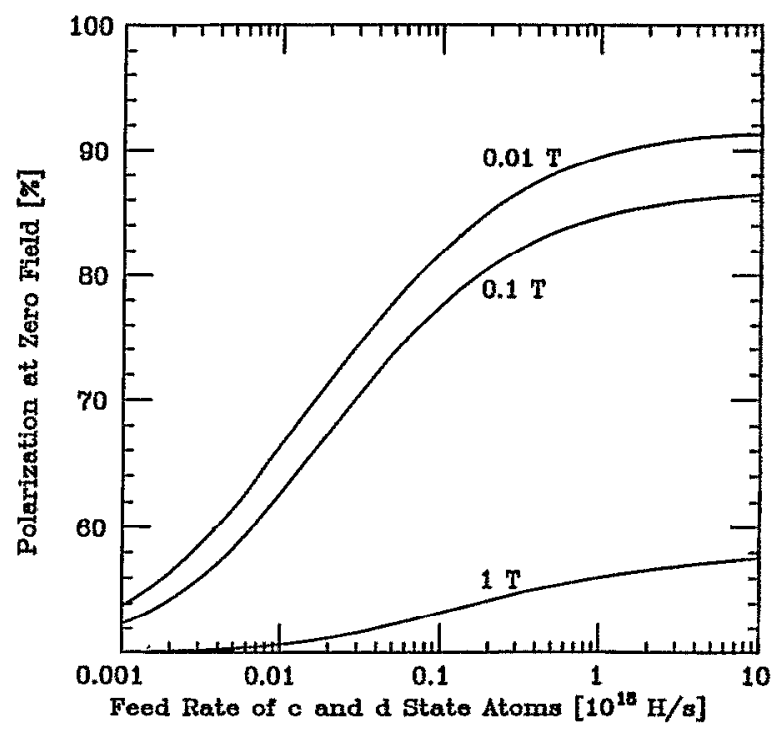

FIG. 3. Spontaneous nuclear polarization at zero magnetic feld of the escaping beam as a function of the feed rate of $c$-and $d$-state atoms. The three curves show the dependence on the magnetic field at the aperture.

accumulated densities $n_{c}$ and $n_{d}$ in front of the exit aperture the two-body spin-exchange rate increases faster than the one-body escape rate.

Solving Eq. (1) for constant state densities typically gives an outcoming flux of $6 \times 10^{17}$ atoms/s and a polarization of $85 \%$ for a feed rate of $10^{18}$ atoms/s and a magnetic field at the aperture of $0.1 \mathrm{~T}$. The heat load to the confining cell, caused by the recombination processes, is 12 $\mathrm{mW}$.

We obtain a very slow $(\sim 0.4 \mathrm{~K})$ nuclear polarized atomic beam which could be focused with a weak sextupole. However, the significant velocity spread of the effusive beam would cause large chromatic aberrations. Berkhout et al. ${ }^{8}$ demonstrated almost perfect quantum reflection of atomic hydrogen from a superfluid-helium surface. Therefore, a superfluid-helium coated parabolic or elliptic mirror, with a smooth surface (roughness less than the thickness of the superfluid helium film) and a focus placed at the exit aperture (see Fig. 1) would create a parallel or a focused beam, respectively. Chromatic aberrations would be avoided and such a method would, therefore, be ideal for focusing the cold effusive beam.

This work was supported by a grant from the U.S. Department of Energy.

'B. W. Statt and A. J. Berlinsky, Phys. Rev. Lett. 45, 2105 (1980).

${ }^{2}$ R. W. Cline, T. J. Greytak, and D. Kleppner, Phys. Rev. Lett. 47, 1195 (1981).

${ }^{3}$ R. Sprik, J. T. M. Walraven, G. N. van Yperen, and I. F. Silvera, Phys. Rev. Lett. 49, 153 (1982).

${ }^{4}$ A. Ya. Katunin, S. A. Vasilyev, I. I. Lukashevich, A. I. Safonov, E. Tjukanov, and S. Jaakkola, Physica B $165 \& 166,743$ (1990).

${ }^{5}$ A. Lagendijk, I. F. Silvera, and B. J. Verhaar, Phys. Rev. B 33, 626 (1986).

${ }^{6}$ H. F. Hess, G. P. Kochanski, T. M. Doyle, N. Masuaza, D. Kleppner, and T. Greytak, Phys. Rev. Lett. 59, 672 (1987).

${ }^{7}$ D. A. Bell, H. F. Hess, G. P. Kochanski, S. Buchman, L. Pollack, Y. M. Xiao, D. Kleppner, and T. J. Greytak, Phys. Rev. B 34, 7670 (1986).

${ }^{8}$ J. J. Berkhout, O. J. Luiten, I. D. Setija, T. W. Hijmans, T. Mizusaki, and J. T. M. Walraven, Phys. Rev. Lett. 63, 1689 (1989). 